\title{
Can Thermal Input from a Prior Universe Account for Relic Graviton Production? Implications for the Cosmological Landscape
}

\author{
Andrew W. Beckwith \\ Physics Department, College of Physics, Chongqing University, Huxi Campus, Chongqing, China \\ Email: Rwill9955b@gmail.com, abeckwith@uh.edu
}

Received 29 April 2016; accepted 4 July 2016; published 8 July 2016

Copyright (C) 2016 by author and Scientific Research Publishing Inc.

This work is licensed under the Creative Commons Attribution International License (CC BY).

http://creativecommons.org/licenses/by/4.0/

cc) (i)

\section{Abstract}

The author presents how to make a link between the low temperature and low entropy of pre big bang state of cosmology as given by Carroll and Chen in 2005, to the quantum cosmology conditions predicted by Weinberg when the temperature reaches $10^{32}$ degrees Kelvin. We do this bridge building in our model construction as a way to get about the fact that cosmological CMB is limited by a red shift about $z=1100$, so in order to get our suppositions consistent with observations, we also examine what happens in our model when we introduce quantization via a shift in values of the Hartle-Hawking wave function from a lower value of nearly zero to one which is set via an upper bound of the Planck's constant of the order of $\mathbf{3 6 0}$ times the square of the Planck's mass.

\section{Keywords}

Relic Graviton Production, Cosmological Landscape

\section{Preliminary Analogy with Regards to Seth Lloyds Universe as a Quantum Computer Paper}

We begin by using the formula given by Seth Lloyd [1] in 2001 with respect to the number of operations the "Universe" can "compute" during its evolution. To begin with, we use formula

$$
\text { \# operations }=\frac{4 E}{\hbar} \cdot\left(t_{1}-\sqrt{t_{1} t_{0}}\right) \approx\left(t_{\text {Final }} / t_{P}\right) \leq 10^{120}
$$


We assume that $t_{1}=$ final time of physical evolution, whereas $t_{0}=t_{P} \sim 10^{-43}$ seconds and that we can set an energy input via assuming in early universe conditions that $N^{+} \neq \varepsilon^{+} \ll 1$, and $0<N^{+}<1$,

$$
E=\left(V_{4-\text { Dim }}\right) \cdot\left[\rho_{\text {Vac }}=\frac{\Lambda}{8 \pi G}\right] \sim N^{+} \cdot\left[\rho_{\text {graviton }} \cdot V_{4 \text {-vol }} \approx \hbar \cdot \omega_{\text {graviton }}\right]
$$
if

Furthermore, if we use the assumption that the temperature is close to $T \approx 10^{29}$ Kelvin initially, we have that

$$
H=\sqrt{8 \pi G \cdot\left[\rho_{\text {crit }} \sim \rho_{\text {graviton }}\right] / 3 \cdot c^{2}} \text { and } \rho_{\text {crit }} \sim \rho_{\text {graviton }} \sim \hbar \cdot \omega_{\text {graviton }} / V_{4-\text { Vol }}
$$

Then

$$
\text { \# operations } \approx 1 /\left[t_{P}^{2} \cdot H\right] \approx \sqrt{V_{4-\mathrm{Vol}}} \cdot t_{P}^{-2} / \sqrt{\left[8 \pi G \hbar \omega_{\text {graviton }} / 3 c^{2}\right]} \approx[3 \ln 2 / 4]^{4 / 3} \cdot\left[S_{\text {Entrophy }} / k_{B} \ln 2\right]^{4 / 3}
$$

So, using the principle of maximum entropy, this leads to certain consequences with regards to the cosmological constant which changes in our model, and initial volume we will elaborate upon in the conclusion.

\section{Introduction to Physical Inputs into This Problem}

First of all we need to consider if there is an inherent fluctuation in early universe cosmology which is linked to a vacuum state nucleating out of "nothing". The answer we have is yes and no.

The vacuum fluctuation leads to production of a dark energy density which we can state is initially due to contributions from an axion wall, which is dissolved during the inflationary era. What we will be doing is to reconcile how that wall was dissolved in early universe cosmology with quantum gravity models, brane world models, and Weinberg's [2] published as of 1972 prediction of a threshold of 10 to the 32 power Kelvin for quantum effects becoming dominant in quantum gravity models. All of this is leading up to conditions in which we can expect relic graviton production which could account for the presence of strong gravitational fields in the onset of Guth style inflation, which would be in line with Penrose's predictions via the Jeans inequality as to low temperature, low entropy conditions for pre inflationary cosmology.

It is noteworthy that Barvinsky et al. [3] in late 2006 recently predicted a range of values of four dimensional Planck's constant values between upper and lower bounds, i.e. this is a way to incorporate the existence of a cosmological constant at about a Planck's time $t_{p}$ with the formation of scale factors which permit the existence of definable space time metrics. A good argument can be made that prior to Planck's time $t_{p}$ that conventional space time metrics, even those adapting to strongly curved space do not apply. Park et al. [4] predict an upper range of cosmological constant values far in excel of Barvinsky [3], and we explain away the difference in terms of a thermal/vacuum energy input into graviton production. We will from now on investigate how this would affect the emergence of an initial state for the scale factor, in the cases where the cosmological constant is first a constant, and then where the cosmological constant parameter is initially far larger.

In order to do this, we are first going to examine how the Friedman equation gives us an evolution of the scale factor $a(t)$, in two cases, Case one will be with a constant cosmological constant. And case two will be when the cosmological constant is far larger than it is today.

Making the case for an initially very, very large cosmological constant will lead to giving a road map into solving the land scape problem. i.e. [3] $\Lambda_{\max }$ (Barvinsky) $=3 m_{p}^{2} / 2 B=360 m_{p}^{2}$ as a peak value, after graviton production would lead to after graviton production would lead to a Hartle-Hawking's universe wave function of the form

$$
\left.\psi_{H H}\right|_{\text {Barvinsky }} \approx \exp \left(-S_{E}\right)=\exp (3 \cdot \pi / 2 \cdot G \Lambda) \neq 0
$$

Whereas, having Parks [4] value for a nearly infinite cosmological constant parameter, due to high temperatures would lead to, prior to graviton production

$$
\left.\psi_{H H}\right|_{\text {Park }} \approx \exp \left(-S_{E}\right)=\exp (3 \cdot \pi / 2 \cdot G \Lambda) \underset{T \rightarrow \infty}{\longrightarrow} 0
$$

This would allow us to make inroads into a solution to the cosmological landscape problem discussed by Guth in 2003 [2] at the Kalvi institute in UC Santa Barbara, i.e. why have $10^{1000}$ or so independent vacuum states as predicted by String theory? 


\section{Statement of the General Problem We Are Investigating}

If one looks at the range of allowed upper bounds of the cosmological constant, we have that the difference between what Barvinsky [3] et al. in late 2006 recently predicted, and Park's upper limit as of 2003, based upon thermal input is a giveaway that a phase transition is occurring at or before Planck's time. This allows for a brief interlude of quintessence.

Begin with assuming that the absolute value of the five dimensional cosmological "constant" parameter is inversely related to temperature, i.e. [4]

$$
\left|\Lambda_{5-\operatorname{dim}}\right| \propto c_{1} \cdot(1 / T)
$$

As opposed to working with the more traditional four dimensional version of the same, minus the minus sign of the brane world theory version [4]

$$
\Lambda_{4-\operatorname{dim}} \propto c_{2} \cdot T
$$

We should note that this is assuming that a release in gravitons occurs which leads to a removal of graviton energy stored contributions to this cosmological parameter which is using a construction from [3]

$$
\Lambda_{4-\text { dim }} \propto c_{2} \cdot T \underset{\text { graviton-production }}{\longrightarrow} 360 \cdot m_{P}^{2} \ll c_{2} \cdot\left[T \approx 10^{32} \mathrm{~K}\right]
$$

Needless to say, right after the gravitons are released one still is seeing a drop off of temperature contributions to the cosmological constant. Then we can write, for small time values $t \approx \delta^{1} \cdot t_{p}, 0<\delta^{1} \leq 1$ and for temperatures sharply lower than $T \approx 10^{12}$ Kelvin

$$
\left(\frac{\Lambda}{\left|\Lambda_{5}\right|}-1\right) \approx O\left(\frac{1}{n}\right) \sim \text { To the order of }(1 / n)
$$

We can do this for length (radii) values proportional to the value of the inverse of what the Hubble parameter is when the absolute value of five dimensional cosmological "constant" parameter is of the order of the four dimensional cosmological "constant" parameter, i.e. when the critical initial nucleation length we consider obeys

$$
L \propto L\left(\Lambda_{4-\operatorname{dim}} \approx\left|\Lambda_{5-\operatorname{dim}}\right|\right) \propto H^{-1}\left(\Lambda_{4-\operatorname{dim}} \approx\left|\Lambda_{5-\operatorname{dim}}\right|\right)
$$

where initially we have temperatures of the order of 1.4 times 10 to the 32 power Kelvin as a thresh hold for the existence of quantum effects. This would pre suppose answering the issue raised by Weinberg [5]. As of 1972, he wrote that for quantum effects to be dominant in cosmology, with a value of critical energy we will use in setting a template for relic graviton production later on.

$$
E_{\text {critical }} \equiv 1.22 \times 10^{28} \mathrm{eV}
$$

This is pre supposing that we have a working cosmology which actually gets to such temperatures at the instance of quantum nucleation of a new universe. Appendix IA. As accessed below gives us a working format as to the dynamics of quantum nucleation as outlined in this article. Appendix $1 \mathrm{~B}$ which is in the same page gives commentary as to temperature dependence of the cosmological constant. Furthermore, Appendix II gives temperature dependence of four and five dimensional versions of a "cosmogical constant". And if there is no temperature dependence, in the $5^{\text {th }}$ dimensional cosmological constant se set as having magnitude $\Lambda$, we still can get a five dimensional line element [6]

$$
\mathrm{d} S_{5-\operatorname{dim}}^{2} \cong \frac{\Lambda \cdot l^{2}}{3} \cdot\{4 \text {-dim Schwartzshield deSitter metric }\}-\mathrm{d} l^{2}
$$

Also Appendix III states what we can expect from a difference in the upper limit of Park's four dimensional inflation value for high temperatures, of the order of 10 to the 32 Kelvin, and the upper bound, Barvinsky [3] predicts, which if put into the Hartle-Hawking's wave function is equivalent to a nucleation-quantization condition which we claim is a way to delineate a solution to the cosmic land scape problem Guth discussed in 2003. Appendix V focuses upon a frequency of relic graviton can be expected, and Also, Appendix IV focuses upon 
why there is a point back in time $t<t_{p}$ (Planck's time interval) where $a(t)$ is chaotic, and reflects a signal discontinuity between inputs of a prior universe and the evolution of today's universe, and Appendix $\mathbf{V}$ gives a criteria as to forming the fraction of a critical density, $\Omega_{G W}=\rho_{G W} / \rho_{C}$ over the logarithmic interval of frequency $\omega$ in a manner which gives criteria for sensitivity goals of $\mathrm{d} \Omega_{G W} / \mathrm{d} \ln \omega \approx 10^{-10}$ or better with the above criteria included above, from R. Brunstein et al.'s 1995 article in Physics Letters B [7]. Finally, and not least is Appendix VI which is considering the dynamics of the Sach-Wolfe effect and CMB. Conventional treatments of the Sach-Wolfe effect often lead to a scaling down of it, via $(1+z)^{-1}$, provided one is using RW space times, as, mentioned by Traschen in 1984 [8]. Also, Appendix VII discusses short term quintessence, which would be a compliment to the Sach-Wolfe effect, and tells us how to link later values of the scale factor with the admittedly wild behavior of the scale factor during inflation.

\section{Weinberg Graviton Production Formula for Relic Gravitons}

As is well known, a good statement about the number of gravitons per unit volume with frequencies between $\omega$ and $\omega+\mathrm{d} \omega$ may be given by (assuming here, that $\bar{k}=1.38 \times 10^{-16} \mathrm{erg} / \mathrm{K}$, and $\mathrm{K}$ is denoting Kelvin temperatures, while we keep in mind that Gravitons have two independent polarization states), as given by Weinberg [2]

$$
n(\omega) \mathrm{d} \omega=\frac{\omega^{2} \mathrm{~d} \omega}{\pi^{2}} \cdot\left[\exp \left(\frac{2 \cdot \pi \cdot \hbar \cdot \omega}{\bar{k} T}\right)-1\right]^{-1}
$$

This formula predicts what was suggested earlier. A surge of gravitons commences due to a rapid change of temperature, i.e. if the original temperature were low, and then the temperature rapidly would heat up.

The hypothesis so presented is that input thermal energy given by the prior universe being inputted into an initial cavity/region dominated by an initially configured low temperature axion domain wall would be thermally excited to reach the regime of temperature excitation permitting an order of magnitude drop of axion density $\rho_{a}$ from an initial temperature $\left.T_{d S}\right|_{t \leq t_{p}} \sim H_{0} \approx 10^{-33} \mathrm{eV}$. We shall, before doing this reference graviton production.

\section{Graviton Power Burst/Where Did the Missing Contributions to the Cosmological "Constant" Parameter Go?}

To do this, we need to refer to a power spectrum value which can be associated with the emission of a graviton. Fortunately, the literature contains a working expression as to power generation for a graviton being produced for a rod spinning at a frequency per second $\omega$, which is by Fontana (2005) [9] at a STAIF new frontiers meeting, which allegedly gives for a rod of length $\hat{L}$ and of mass $m$ a formula for graviton production power [9]

$$
P(\text { power })=2 \cdot \frac{m_{\text {graviton }}^{2} \cdot \hat{L}^{4} \cdot \omega_{\text {net }}^{6}}{45 \cdot\left(c^{5} \cdot G\right)}
$$

The point is though that we need to say something about the contribution of frequency needs to be understood as a mechanical analogue to the brute mechanics of graviton production. We can view the frequency $\omega_{\text {net }}$ as an input from an energy value, with graviton production number (in terms of energy) as given approximately via an integration of Equation (14) above, $\widehat{L} \propto l_{P}$, mass $m_{\text {graviton }} \propto 10^{-60} \mathrm{~kg}$. It also depends upon a HUGE number of relic gravitons being produced, due to the temperature variation so proposed.

$$
\langle n(\omega)\rangle=\frac{1}{\omega \text { (net value) })} \int_{\omega 1}^{\omega 2} \frac{\omega^{2} \mathrm{~d} \omega}{\pi^{2}} \cdot\left[\exp \left(\frac{2 \cdot \pi \cdot \hbar \cdot \omega}{\bar{k} T}\right)-1\right]^{-1}
$$

And then one can set a normalized "energy input" as $E_{\text {eff }} \equiv\langle n(\omega)\rangle \cdot \omega \equiv \omega_{\text {eff }}$; with $\hbar \omega \underset{\hbar \equiv 1}{\longrightarrow} \omega \equiv\left|E_{\text {critical }}\right|$ being given in Equation (10) above, which leads to the following Table 1 of results, with $T^{*}$ being an initial temperature of the pre inflationary universe condition.

The outcome is that there is a distinct power spike associated with Equation (9) and Equation (10), which is congruent with a relic graviton burst. Our next task will be to configure the conditions via brane world dynamics leading to graviton production. 
Table 1. How to outline the existence of a relic graviton burst.

\begin{tabular}{ccc}
\hline N1 $=1.794 \mathrm{E}-6$ for & Temp $=T^{*}$ & Power $=0$ \\
N2 $=1.133 \mathrm{E}-4$ for & Temp $=2 T^{*}$ & Power $=0$ \\
N3 $=7.872 \mathrm{E}+21$ for & Temp $=3 T^{*}$ & Power $=1.058 \mathrm{E}+16$ \\
N4 $=3.612 \mathrm{E}+16$ for & Temp $=4 T^{*}$ & Power $\cong$ very small value \\
N5 $=4.205 \mathrm{E}-3$ for & Temp $=5 T^{*}$ & Power $=0$ \\
\hline
\end{tabular}

To build the Kaluza-Klein theory, one picks an invariant metric on the circle $S^{1}$ that is the fiber of the $U$ (1)-bundle of electromagnetism. This leads to construction of a two component scalar term with contributions of different signs. i.e. [10]

$$
S_{5}=-\int \mathrm{d}^{4} x \cdot V_{\text {eff }}\left(R_{\text {phys }}(x)\right) \rightarrow-\int \mathrm{d}^{4} x \cdot \tilde{V}_{\text {eff }}\left(R_{\text {phys }}(x)\right)
$$

We should briefly note what an effective potential is in this situation [10].

We get

$$
\tilde{V}_{\text {eff }}\left(R_{\text {phys }}(x)\right)=\frac{K^{2}}{2 \cdot m_{5}} \cdot \frac{1+\exp \left(m_{5} \cdot \pi \cdot R_{\text {phys }}(x)\right)}{1-\exp \left(m_{5} \cdot \pi \cdot R_{\text {phys }}(x)\right)}+\frac{\tilde{K}^{2}}{2 \cdot \tilde{m}_{5}} \cdot \frac{1-\exp \left(\tilde{m}_{5} \cdot \pi \cdot R_{\text {phys }}(x)\right)}{1+\exp \left(\tilde{m}_{5} \cdot \pi \cdot R_{\text {phys }}(x)\right)}
$$

This above system has a metastable vacuum for a given special value of $R_{\text {phys }}(x)$. Start with [10]-[13]

$$
\begin{gathered}
\Psi \propto \exp \left(-\int \mathrm{d}^{3} x_{\text {space }} \mathrm{d} \tau_{\text {Euclidian }} L_{E}\right) \equiv \exp \left(-\int \mathrm{d}^{4} x \cdot L_{E}\right) \\
L_{E} \geq|Q|+\frac{1}{2} \cdot\left(\tilde{\phi}-\phi_{0}\right)^{2}\{\} \underset{Q \longrightarrow 0}{\longrightarrow} \frac{1}{2} \cdot\left(\tilde{\phi}-\phi_{0}\right)^{2} \cdot\{\}
\end{gathered}
$$

Part of the integrand in Equation (20) is known as an action integral [9], $S=\int L \mathrm{~d} t$, where L is the Lagrangian of the system. Whereas we also are assuming a change to what is known as Euclidean time, via $\tau=i \cdot t$, which has the effect of inverting the potential to emphasize the quantum bounce hypothesis of Sidney Coleman. In that hypothesis, $L$ is the Lagrangian with a vanishing kinetic energy contribution, i.e. $L \rightarrow V$, where $V$ is a potential whose graph is "inverted" by the Euclidian time. Here, the spatial dimension $R_{\text {phys }}(x)$ is defined so that [10]-[12]

$$
\tilde{V}_{\text {eff }}\left(R_{\text {phys }}(x)\right) \approx \text { constant }+1 / 2 \cdot\left(R_{\text {phys }}(x)-R_{\text {critical }}\right)^{2} \propto \tilde{V}_{2}(\tilde{\phi}) \propto \frac{1}{2} \cdot\left(\tilde{\phi}-\phi_{C}\right)^{2}
$$

And using the method [13] [14]

$$
\{\}=2 \cdot \Delta \cdot E_{\text {gap }}
$$

We should note that the quantity \{\}$=2 \cdot \Delta \cdot E_{\text {gap }}$ referred to above has a shift in minimum energy values between a false vacuum minimum energy value, $E_{\text {false min, }}$ and a true vacuum minimum energy $E_{\text {true min }}$, with the difference in energy reflected in Equation (16) above. While noting Equation (22) is straight from [14].

This requires,

$$
\tilde{V}_{\text {eff }}\left(R_{\text {phys }}(x)\right) \approx \text { Constant }+1 / 2 \cdot\left(R_{\text {phys }}(x)-R_{\text {critical }}\right)^{2} \propto V_{0}+\frac{m}{2} \cdot\left[\phi-\varphi_{\text {fluctuations }}\right]_{\text {-dim }}^{2}
$$

So that one can make equivalence between the following statements. These need to be verified via serious analysis.

$$
\begin{gathered}
\text { Constant } \leftrightarrow V_{0} \\
1 / 2 \cdot\left(R_{\text {phys }}(x)-R_{\text {critical }}\right)^{2} \leftrightarrow \frac{m}{2} \cdot\left[\phi-\varphi_{\text {fluctuations }}\right]_{4-\text { dim }}^{2}
\end{gathered}
$$

Proof of Claim: There is a way, for finite temperatures for defining a given four-dimensional cosmological constant. Check Appendix II below in working with these values, we should pay attention to how $\cdot \Lambda_{4-\text { dim }}$ is 
defined by Park, et al. [4] with $\varepsilon^{*}=\frac{U_{T}^{4}}{k^{*}}$ and $U_{T} \propto($ external temperature $)$, and $k^{*}=\left(\frac{1}{\text { 'AdS curvature }}\right)$ so that by [4]

$$
\begin{gathered}
\cdot \Lambda_{4-\text { dim }}=8 \cdot M_{5}^{3} \cdot k^{*} \cdot \varepsilon^{*} \underset{\text { external temperature } \rightarrow 3 \text { Kelvin }}{\longrightarrow}(0.0004 \mathrm{eV})^{4} \\
\left.\Delta \Lambda_{\text {total }}\right|_{\text {effective }}=\lambda_{\text {other }}+\Delta V \underset{\Delta V \rightarrow \text { end chaotic inflation potential }}{\longrightarrow} \Lambda_{\text {observed }} \cong \Lambda_{4-\text { dim }}(3 \text { Kelvin })
\end{gathered}
$$

\section{Detection of Gravitons as Spin 2 Objects via Available Detector Systems?}

First of all, we will state that in describing Gravitons, we are not precluding what t'Hooft said about a possibly necessary Deterministic foundation to quantum physics. The discussion so engendered is to help parse the likelihood that the conjecture in [15] has merit. But to do so, we should determine if the entire idea of.

To briefly review what we can say now about standard graviton detection schemes, as mentioned above, Rothman wrote Dyson [16] [17] doubts we will be able to detect gravitons via present detector technology. The conundrum is that if one defines the criterion for observing a graviton as [16]

$$
\frac{f_{\gamma} \cdot \sigma}{4 \cdot \pi} \cdot\left(\frac{\alpha}{\alpha_{g}}\right)^{3 / 2} \cdot \frac{M_{s}}{R^{2}} \cdot \frac{1}{\varepsilon_{\gamma}} \geq 1
$$

Here,

$$
f_{\gamma}=\frac{L_{\gamma}}{L}
$$

This has $f_{\gamma} \approx \breve{L}_{\gamma} / \breve{L}$ as a graviton sources luminosity divided by total luminosity and $R$ as the distance from the graviton source, to a detector. Furthermore, $\alpha=e^{2} / \hbar$ and $\alpha_{g}=G m_{p}^{2} / \hbar$ a constant while $\varepsilon_{\gamma}$ is the graviton potential energy. Here, $\breve{L}_{\gamma}$-luminosity of graviton producing process $\geq 7.9 \times 10$ to the 14 th ergs/s, while $\breve{L}$-general background luminosity which is usually $\gg L_{\gamma}$. At best, we usually can set $f_{\gamma}=0.02$, which does not help us very much. That means we need to look elsewhere than the usual processes to get satisfaction for Graviton detection. This in part is why we are looking at relic graviton production for early universe models, usually detectable via the criteria developed for white dwarf stars of one graviton for $10^{13-14}$ neutrinos.

We should state that we will generally be referring to a cross section which is frequently the size of the square of Planck's length $l_{p}$ which means we really have problems in detection, if the luminosity is so low. An upper bound to the cross section $\sigma$ for a graviton production process $\approx 1 / M$ with $M$-Planck scale in $4+n$ dimensions $\equiv\left(M_{P}^{2} / \hat{V}_{n}\right)^{1 / 2+n}$, and this is using a very small $\hat{V}_{n}$-Compactified early universe extra dimension "square" volume $\approx 10$ - $15 \mathrm{~mm}$ per side.

As stated in the manuscript, the problem then becomes determining a cross section $\sigma$ for a graviton production process and $f_{\gamma}=L_{\gamma} / L$. Here, a 4-dimensional graviton emission cross section goes like $1 / \mathrm{M}$. The existence of branes is relevant to graviton production. And understanding the link between the brane world prediction of relic graviton production and Wheelers numerical model is crucial to understanding more of the origins of the quantum geometry problem of early universe conditions.

\section{Conclusions}

So far, we have tried to reconcile the following.

First is that Brane world models will not permit Akshenkar's quantum bounce [18] [19]. The quantum bounce idea is used to indicate how one can reconcile axion physics with the production of dark matter/dark energy, later on in the evolution of the inflationary era where one sees Guth style chaotic inflation for times $t \geq t_{p}$ and the emergence of dark energy during the inflation era.

In addition, we need to remember that references [20] [21] are predicting a lower temperature, and low initial entropy state prior to the big bang. This is a detail which needs to be reviewed so as to keep consistency in our cosmological model building. We need to not forget the radical nature of a build-up of temperature to the Kelvin temperature threshold of $10^{32}$ degrees Kelvin at the start of the big bang, and make certain that our bridge from 
pre big bang states, with presumably no quantum gravity, is not inconsistent with what happens at $10^{32}$ degrees Kelvin, which is mentioned as the onset of quantum gravity effects in cosmology. How does one ramp up to the high energy values greater than temperatures $10^{12}$ Kelvin during nucleosynthesis? The solution offered is novel and deserves further inquiry and investigation.

Thirdly is the issue of relic graviton production. How to observe it? The last section about limitations of graviton detectors, as opposed to gravity waves, points to the obvious problems. Current estimates speak of a detector system of the size of Jupiter to be able to detect a single graviton. This is patently absurd and needs to be addressed, likely by coupling graviton production with neutrino physics.

Now for suggestions as to future research. We are in this situation making reference to solve the cosmological "constant" problem without using G. Gurzadyan and She-Sheng Xue’s [22] approach which is fixed upon the scale factor $a(t)$ for a present value of the cosmological constant. This among other things is to reconcile changing values of the cosmological constant with the enormous value of the critical energy needed as a threshold value for quantum gravity effects. Namely

$$
E_{\text {critical }} \equiv 1.22 \times 10^{28} \mathrm{eV}
$$

One of our challenges ahead will be to link the onset of graviton production as indicated above, with the critical threshold energy, which we assert is a trigger for graviton production. In doing this we will be looking, maybe via the Sach-Wolfe effect, evidences for not only the higher frequency range of Graviton radiation, but also experimental evidence for the existence of short term quintessence, as outlined in Appendix VII in this paper. Furthermore, looking at Equation (4) of the first page, we would have a dramatically lowered value for a net range of graviton frequencies if the initial volume of space for graviton production is localized in the regime near the Planck time interval, i.e. we may need to, for information theory reasons go out to the $z \approx 1100$ red shift limit after 1000 years after the big bang to commence a region of space consistent with Equation (4) of the first page, with high net graviton frequencies. This requires that we are assuming an initially large value for the cosmological constant.

We have the paradoxical result that we may need a huge influx of gravitons to give the initially low temperature, low entropy initial conditions given by J. Chen, and Sean Carroll in [20] [21] and the initially low temperature conditions changed by a gigantic cosmological constant, which after graviton production would lead to Barvinsky's lower value of the maxim value of 360 times the square of Planck's mass to give us an answer to the cosmological constant problem, i.e. The Hartle-Hawking wave function comes into being right AFTER graviton production, whereas it is zero initially beforehand. This would lead to a favored state as to the nucleation of the cosmological landscape due to changes in the cosmological constant at or before a Planck's time interval, where we would have $z \sim 10^{26}$, whereas we would have to go out to the region of space where $z \approx 1100$ to be consistent with regards to Seth Lloyds' [1] measurement of the computational space time limits of how the universe evolves in time.

Finally, the chaotic conditions given in Appendix IV argue for a signal/causal relationship discontinuity between a prior universe, and our present universe, as it is evolving.

Reference [23] is the traditional way to handle the matter of causal structure. We claim in our document that it is not guaranteed and that it is debatable (causal structure continuity).

So we study it and this also ties into the question if we have classical versus quantum conditions at the start of cosmological evolution.

In doing so, we need to also consider the possibility of getting more information about the quantum versus classical nature of (signal) waveforms representing gravitational waves from our work, and this in lieu of [24] which is due to binary black hole generation of GW at a frequency range of 35 to $250 \mathrm{~Hz}$ with a peak gravitational-wave strain of $1.0 \times(10$ to the -21 power). As stated by [24] the LIGO findings match the waveform predicted by general relativity for the in spiral and merger of a pair of black holes and the ring down of the resulting single black hole. We next need to build on our own techniques, to determine if the results of [24] are comparable with relic gravitational waves, and what this says as far as detection characteristics. In addition, the causal discontinuity as alluded to in, should be seen to be a way to delve into what Corda in [25] [26] brought up. i.e. if there is a third polarization of Gravitation, and if so do we need to examine Scalar-tensor theories of Gravity, or if GR is necessary and sufficient for explaining the observed in the early universe for the genesis of Gravitation. Note that [27] has also some considerations as to massive gravity which are intertwined with the issue brought 
up by Corda in [25] [26].

\section{Acknowledgements}

This work is supported in part by National Nature Science Foundation of China grant No. 11375279.

\section{References}

[1] Lloyd, S. (2001) Computational Capacity of the Universe. http://arxiv.org/abs/quant-ph/0110141

[2] Weinberg, S. (1972) Gravitation and Cosmology: Principles and Applications of the General Theory of Relativity. John Wiley and Sons, Inc., New York.

[3] Barvinsky, K. and Yu, A. (2006) Thermodynamics from Nothing: Limiting the Cosmological Constant Landscape. Physical Review D, 74, 121502.

[4] Park., D.K., Kim, H. and Tamarayan, S. (2002) Nonvanishing Cosmological Constant of Flat Universe in Brane-World Senarios. http://arxiv.org/abs/hep-th/0111081

[5] Guth, A.H. (2003) Eternal Inflation. http://online.itp.ucsb.edu/online/strings_c03/guth/pdf/KITPGuth_2.pdf

[6] Wesson, P.S. (2000) Space-Time-Matter: Modern Kaluza-Klein Theory. World Press Scientific, P.t.e., Ltd., Singapore.

[7] Brustein, R. and De Alwis, S.P. (1990) Non-Perturbative Divergence in Critical String Theory. Physics Letters B, 247, 31-35. http://dx.doi.org/10.1016/0370-2693(90)91044-C

[8] Traschen, J. (1984) Causal Cosmological Perturbations and Implications for the Sachs-Wolfe Effect. Physical Review D, 29, 1563. http://dx.doi.org/10.1103/PhysRevD.29.1563

[9] Fontana, G. (2005) Gravitational Wave Propulsion. In: El-Genk, M.S., Ed., CP746, Space Technology and Applications International Forum-STAIF, American Institute of Physics, Melville.

[10] Sundrum, R., (2005) Extra Dimensions. SLAC Summer Institute: Gravity in the Quantum World and the Cosmos. http://www-conf.slac.stanford.edu/ssi/2005/lec_notes/Sundrum1/sundrum1.pdf

[11] Buusso, R. and Randall, L. (2001) Holographic Domains of Anti-de Sitter Space. https://arxiv.org/abs/hep-th/0112080

[12] Lee, J. and Sorkin, R.D. (1988) Derivation of a Bogomolnyi Inequality In Five Dimensional Kaluza-Klein Theory. Communications in Mathematical Physics, 116, 353-364. http://dx.doi.org/10.1007/BF01229199

[13] Zee, A. (2003) Quantum Field Theory in a Nutshell. Princeton University Press, Princeton, 279-280.

[14] Beckwith, A.W. (2006) An Open Question: Are Topological Arguments Helpful In Setting Initial Conditions For Transport Problems in Condensed Matter Physics. Modern Physics Letters B, 20, 233-243. http://dx.doi.org/10.1142/s0217984906010585

[15] Hooft, G. (2002) Determinism beneath Quantum Mechanics. http://arxiv.org/PS_cache/quant-ph/pdf/0212/0212095v1.pdf

[16] Rothman, T. and Boughn, S. (2006) Can Gravitons Be Detected? Foundations of Physics, 36, 1801-1825. http://arxiv.org/abs/gr-qc/0601043

[17] Dyson, F. (2012) Is a Graviton Detectable? http://publications.ias.edu/sites/default/files/poincare2012.pdf

[18] Ashtekar, A., Pawlowski, T. and Singh, P. (2006) Quantum Nature of the Big Bang. Physical Review Letters, 96, 141301. http://dx.doi.org/10.1103/PhysRevLett.96.141301

[19] Ashtekar, A., Pawlowski, T. and Singh, P. (2006) Quantum Nature of the Big Bang: An Analytical and Numerical Investigation. Physical Review D, 73, 124038. http://arxiv.org/abs/gr-qc/0604013

[20] Carroll, S.M. and Chen, J. (2005) Does Inflation Provide Natural Initial Conditions for the Universe? International Journal of Modern Physics D, 14, 2335-2340. http://arxiv.org/abs/gr-qc/0505037

[21] Carroll, S.M. and Chen, J. (2004) Spontaneous Inflation and the Origin of the Arrow of Time. http://arxiv.org/abs/hep-th/0410270

[22] Gurzadyan, G. and Xue, S.-S. (2003) On the Estimation of the Current Value of the Cosmological Constant. Modern Physics Letters A, 18, 561-568.

[23] Dowker, F. (2005) Causal Sets and the Deep Structure of Space-Time. http://arxiv.org/abs/gr-qc/0508109

[24] Abbott, B.P., et al. (2016) Observation of Gravitational Waves from a Binary Black Hole Merger. Physical Review Letters, 116, 061102. https://physics.aps.org/featured-article-pdf/10.1103/PhysRevLett.116.061102

[25] Corda, C. (2008) Massive Gravitational Waves from the $\mathrm{R}^{2}$ Theory of Gravity: Production and Response of Interferometers. International Journal of Modern Physics A, 23, 1521-1535. http://dx.doi.org/10.1142/S0218271809015904 
[26] Corda, C. (2009) Interferometric Detection of Gravitational Waves: The Definitive Test for General Relativity. International Journal of Modern Physics D, 18, 2275-2282. http://arxiv.org/abs/0905.2502 http://dx.doi.org/10.1142/S0218271809015904

[27] Beckwith, A. (2016) Gedanken Experiment for Fluctuation of Mass of a Graviton, Based on the Trace of GR Stress Energy Tensor-Pre Planckian Conditions that Lead to Gaining of Graviton Mass, and Planckian Conditions That Lead to Graviton Mass Shrinking to $10^{-62}$ Grams. Journal of High Energy Physics, Gravitation and Cosmology, 2, 19-24. http://dx.doi.org/10.4236/jhepgc.2016.21002

[28] Beckwith, A. (2016) Does a Randall-Sundrum Brane World Effective Potential Influence Axion Walls Helping to Form a Cosmological Constant Affecting Inflation? Journal of High Energy Physics, Gravitation and Cosmology, 2, 125-153. http://dx.doi.org/10.4236/jhepgc.2016.21013

[29] Padmanabhan, T. (2005) 100 Years of Relativity, Space-Time Structure: Einstein and Beyond. World Scientific Publishing Co. Pte. Ltd., Singapore, 175-201.

[30] Puthoff, H. (2009) Private Communications at the Institute of Advanced Study, in 2009, in Austin, Texas in a Visit to the Center, as a Guest of Dr. Eric Davis.

[31] Dodelson, S. (2003) Modern Cosmology. Academic Press, Cambridge.

[32] Chen, P. (1995) Resonant Photon-Graviton Conversion and Cosmic Microwave Background Fluctuations. Physical Review Letters, 74, 634-637. http://dx.doi.org/10.1103/PhysRevLett.74.634 


\section{Appendix IA: First Table with Respect to Phenomenology}

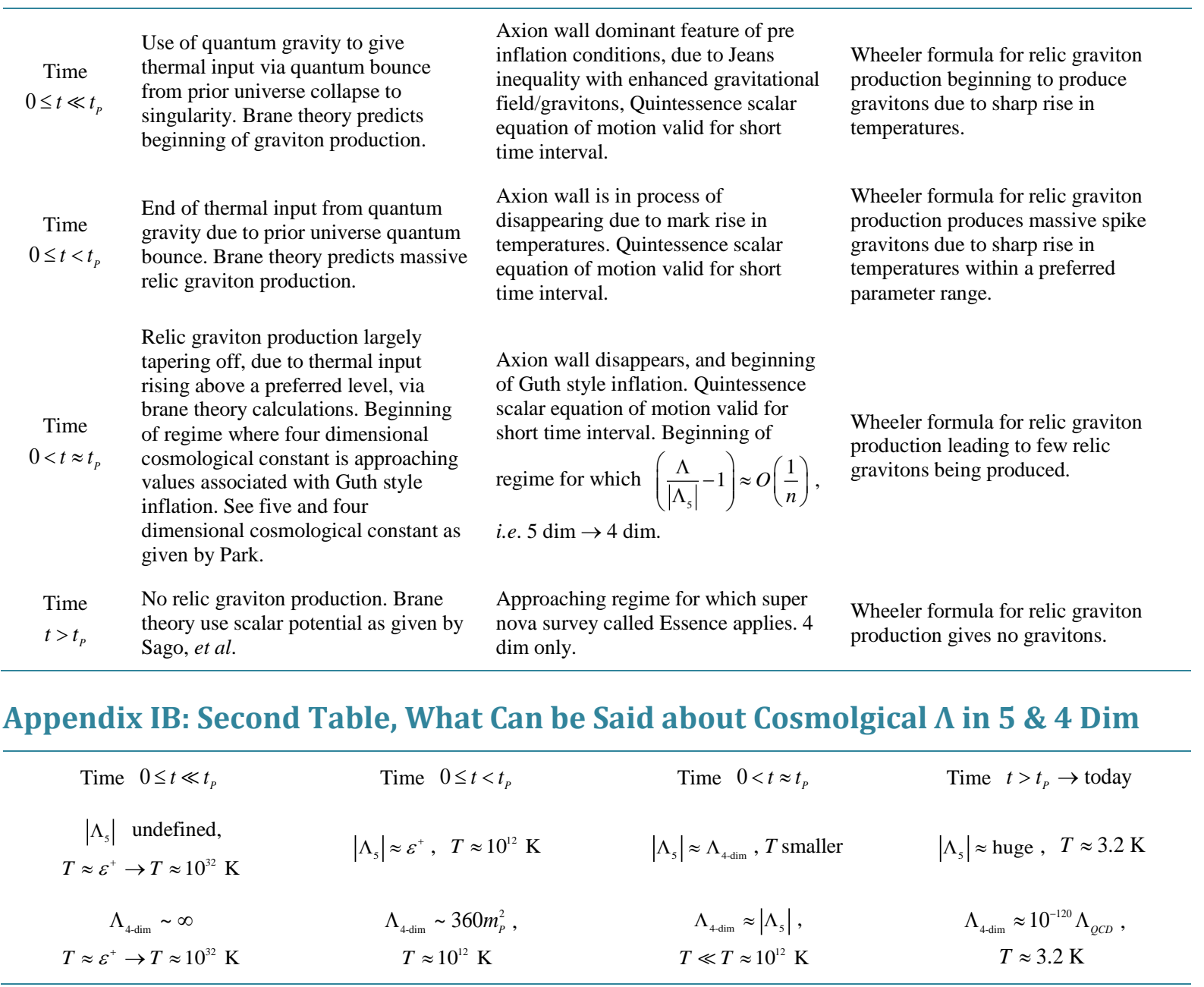

\section{Appendix II: How to Define a Temperature Dependent Four and Five Dimensional Cosmological Constant Parameter}

We define, via Park’s article [4]

$$
k^{*}=\left(\frac{1}{\text { 'AdS curvature }}\right)
$$

Park et al. note that if we have a "horizon" temperature term

$$
U_{T} \propto \text { (external temperature) }
$$

We can define a quantity

$$
\varepsilon^{*}=\frac{U_{T}^{4}}{k^{*}}
$$

Then there exists a relationship between a four-dimensional version of the $\Lambda_{\text {eff }}$, which may be defined by noting

$$
\Lambda_{5-\operatorname{dim}} \equiv-3 \cdot \Lambda_{4-\operatorname{dim}} \cdot\left(\frac{U_{T}}{k^{* 3}}\right)^{-1} \propto-3 \cdot \Lambda_{4-\operatorname{dim}} \cdot\left(\frac{\text { external temperature }}{k^{* 3}}\right)^{-1}
$$


So

$$
\Lambda_{5-\text { dim }} \underset{\text { external temperature } \rightarrow \text { small }}{\longrightarrow} \text { Large value }
$$

And set

$$
\left|\Lambda_{5-\text { dim }}\right|=\Lambda_{\text {eff }}
$$

This is important because of the datum that the defined an initial peak value of $\Lambda_{4 \text {-dim }} \sim \infty$ as we saw an increase of temperature $T \approx \varepsilon^{+} \rightarrow T \approx 10^{32} \mathrm{~K}$ having a nearly zero value five dimensional cosmological parameter which subsequently becomes huge, as $\cdot \Lambda_{4-\mathrm{dim}}=8 \cdot M_{5}^{3} \cdot k^{*} \cdot \varepsilon^{*} \underset{\text { external temperature } \rightarrow 3 \mathrm{Kelvin}}{\longrightarrow}(0.0004 \mathrm{eV})^{4}$ as we approach current cosmological conditions when $T \approx 3 \mathrm{~K}$.

\section{Appendix III: How a Difference in Values of the Cosmological Constant, as Given by Park et al. (2003) and Barvinsky et al. (2006) Implies Quantization of an Initial Vacuum State via Different Initial Conditions of the Hartle-Hawking Wave Function}

In order to reference this argument, it is useful to note that Barvinsky [3] in late 2006 came up with an upper value of the cosmological constant as given by:

$$
\left.\Lambda_{\text {max }}\right|_{\text {Barvinsky }} \cong 360 \cdot m_{P}^{2}
$$

And a minimum value of

$$
\left.\Lambda_{\text {min }}\right|_{\text {Barvinsky }} \cong 8.99 \cdot m_{P}^{2}
$$

In contrast to the nearly infinite value of the Planck's constant as given by Park et al. (2003).

$\cdot \Lambda_{4 \text {-dim }}$ is defined by Park, et al. [4] with $\varepsilon^{*}=\frac{U_{T}^{4}}{k^{*}}$ and $U_{T} \propto$ (external temperature), and $k^{*}=\left(\frac{1}{\text { 'AdS curvature }}\right)$ so that

$$
\left.\Lambda_{4-\text { dim,Max }}\right|_{\text {Park }} \underset{T \mapsto 10^{32} \text { Kelvin }}{\longrightarrow} \infty
$$

As opposed to a minimum value of

$$
\cdot \Lambda_{4-\text { dim }}=8 \cdot M_{5}^{3} \cdot k^{*} \cdot \varepsilon^{*} \underset{\text { external temperature } \rightarrow 3 \text { Kelvin }}{\longrightarrow}(0.0004 \mathrm{eV})^{4}
$$

When applied w.r.t. to the Hartle-Hawking wave function of [3]

$$
\psi_{H H} \approx \exp \left(-S_{E}\right)=\exp (3 \cdot \pi / 2 \cdot G \Lambda)
$$

We will observe having

$$
\left.\psi_{H H}\right|_{\Lambda(\text { Park })} \cong \varepsilon^{+} \propto 0^{+}
$$

As opposed to a nucleated value of

$$
\left.\left.\psi_{H H}\right|_{\Lambda(\text { Barvinsky })} \sim \exp \left(3 \cdot \pi / 2 \cdot G \cdot 360 \cdot m_{P}^{2}\right) \gg \psi_{H H}\right|_{\Lambda(\text { Park })} \cong \varepsilon^{+} \propto 0^{+}
$$

De facto, the dissolving of axion walls alluded to by when we have a thermal input with back ground temperatures at or greater than $10^{32}$ Kelvin we are observing for $0<\varepsilon^{+} \ll 1$ and times $t \ll t_{P}$

$$
f\left[m_{\text {axion }}(T)\right]_{T \approx 0^{32} \text { Kelvin }} \propto O\left(\left(\varepsilon^{+}\right) \cdot m^{2}\right)
$$

Is consistent with temperatures which are nearly infinite, which is alluded to by Barvinsky's temperature scaling of [4]

$$
T(\text { temp }) \cong H / \pi \cdot \sqrt{1-2 \cdot B \cdot H} \underset{H \rightarrow 1 / \sqrt{2 \cdot B}}{\longrightarrow} T(\text { max-temp }) \geq 10^{32} \text { Kelvin }
$$


Where we have a scaled Hubble value of

$$
H_{k}^{2} \cong \frac{1}{2 B} \cdot\left(1-\frac{\ln ^{2} k^{2}}{2 k^{2} \pi^{2}}\right)
$$

and Planck's constant as given by

$$
\Lambda_{\max }(\text { Barvinsky })=3 m_{P}^{2} / 2 B=360 m_{P}^{2}
$$

This correlates well with Carroll's estimated value of a peak gravitational frequency value of , when Temerature [20] [21] is about 10 to the 32 Kelvin greater than or equal to the electro weak temperature $T \sim 200 \mathrm{GeV}$. $f_{\text {peak }} \sim 10^{-3} \cdot\left(\frac{T}{1000 \mathrm{GeV}}\right) \mathrm{Hz} \sim N * 10^{-3} \mathrm{~Hz}$ which can be observed by LISA. If one Kelvin is about 1.16 times $10^{13} \mathrm{GeV}$, then having a temperature of the order of $10^{32}$ Kelvin means a frequency range of $f_{\text {peak }} \sim 10^{-3} \cdot\left(\frac{10^{32} \mathrm{GeV}}{1000 \mathrm{GeV}}\right) \mathrm{Hz} \sim 10^{32} \mathrm{~Hz}$. This is what is could be expected at the peak Park value of the cosmological constant, as outlined.

\section{Appendix IV: How the Evolution of the Scale Factor Is Influenced by Initial Choices of the "Cosmological Constant" Parameter at the Onset of Inflation}

We will begin with an analysis of the Friedman equation and how the information about an alleged phase transition alluded to in Appendix IV above affects the evolution of the scale factor $a(t)$. It is my contention, partly based upon the change in the cosmological constant at or about Planck's time interval $t_{p}$, combined with the shift in the Hawkings-Hartle wave [3] function at about the same time, from essentially a zero value to a non zero measurable quantity, that the scale factor

$$
(\dot{a} / a)^{2}=\frac{8 \pi G}{3} \cdot\left[\rho_{\text {rel }}+\rho_{\text {matter }}\right]+\frac{\Lambda}{3}
$$

With

$$
\rho_{\text {rel }} \equiv\left(\frac{a_{\text {present-era }}}{a(t)}\right)^{4} \cdot\left(\rho_{\text {rel }}\right)_{\text {present-era }}
$$

And

$$
\rho_{m} \equiv\left(\frac{a_{\text {present-era }}}{a(t)}\right)^{3} \cdot\left(\rho_{m}\right)_{\text {present-era }}
$$

This leads to a largely undefined statement about forming a consistent statement about scale expansion factors

$$
V \approx(1 / n) \cdot \phi^{n}
$$

involves a change of scale factor of the form [28] [29]

$$
a(\phi(t)) \cong a_{\text {init }} \cdot \exp \left(\frac{4 \cdot \pi}{n} \cdot\left(\phi_{\text {init }}^{2}-\phi^{2}(t)\right)\right)
$$

We take into account the axion type physics given in [28] but Equation (5) is straight from [29].

The first hint of trouble can be seen when considering when the axion walls as mentioned in our model disappear,

$$
a_{\text {init }} \cdot \exp \left(\frac{4 \cdot \pi}{n} \cdot\left(\phi_{\text {init }}^{2}-\phi^{2}(t)\right)\right) \underset{\text { AXION } \rightarrow 0}{\longrightarrow} a_{\text {init }} \cdot \exp \left(\frac{4 \cdot \pi}{2} \cdot\left(\phi_{\text {init }}^{2}-\phi^{2}(t)\right)\right)
$$

But before that we need to understand if or not we can even define what happens if and when

$$
(\dot{a} / a)^{2}=N_{\text {Planck }}<\infty
$$


This is in a largely instantaneous period of time. The guess we are offering is that this discontinuity, largely eliminates being able to analyze the evolution of a scale factor.

The two equations we are going to work with are as follows. First of all, we will consider what we can do with when we do not consider a constant $\dot{H}$ in the following equation. In that case Equation (8) below is an integro differential equation given below

$$
\left[\frac{\dot{a}}{a}\right]^{2}=\frac{\ddot{a}}{a}-\dot{H}=\frac{\Lambda \cdot a^{2}}{3}+\left(\frac{8 \pi}{3}\right) \cdot\left[\frac{\left(\rho_{\text {rel }}\right)_{0} a_{0}^{4}}{a^{2}(t)}+\frac{\left(\rho_{m}\right)_{0} a_{0}^{3}}{a(t)}\right]
$$

This leads us to a highly non linear integral equation for evaluation of the scale factor as follows

$$
\int \mathrm{d} a \frac{1}{\left[a^{4}(t)+\frac{8 \pi}{\Lambda}\left[\left(\rho_{\text {rel }}\right)_{0} a_{0}^{4}+\left(\rho_{m}\right)_{0} a_{0}^{3} a(t)\right]\right]^{1 / 2}} \equiv \sqrt{\frac{\Lambda}{3}} \cdot \int \mathrm{d} t
$$

The second case we will consider will be what if we consider a constant negative $\dot{H}$ leading to the following differential equation to work with:

$$
\ddot{a}+|\dot{H}| a=\frac{\Lambda \cdot a^{3}}{3}+\left(\frac{8 \pi}{3}\right) \cdot\left[\frac{\left(\rho_{\text {rel }}\right)_{0} a_{0}^{4}}{a(t)}+\left(\rho_{m}\right)_{0} a_{0}^{3}\right]
$$

We wish to note that in analyzing both Equation (9) above, and Equation (10) above we will be looking at different values of the cosmological factor $\Lambda$. In the case that both the $|\dot{H}|$ and $\Lambda$ are not small, Equation (10) above exhibits extraordinarily striking physical phenomenology. We wish to also state that when we have small values for the scalar factor $a(t)$ as well as not overly large $\Lambda$ values, that Equation (9) above allows us to retrieve standard cosmological scaling of $a(t) \sim t^{\lambda}$ for $1 / 2<\lambda \leq 1$, whereas not putting a small value upon the upper bound of the scale factor $a(t)$ allows for an extremely non linear polynomial equation for $u \equiv 1 / a(t)$ which has striking implications upon the evolution of the scale factor. We shall first of all analyze Equation (9) above with respect to different values of $\Lambda$ and the scale factor $a(t)$. Starting off with analyzing Equation (9) above means using the following expansion in Equation (9) above again and again.

$$
(1+x)^{-1 / 2}=1-\frac{x}{2}+\frac{1 \times 3}{2 \times 4} \cdot x^{2}-\frac{1 \times 3 \times 5}{2 \times 4 \times 6} \cdot x^{3}+\cdots
$$

This means we can make the following approximations to Equation (9) above: If $u=a^{-1}$

$$
\sqrt{\frac{\Lambda}{3}} \cdot \int \mathrm{d} t \equiv-\int \frac{\mathrm{d} u}{\sqrt{1+\frac{8 \pi}{\Lambda}\left[\left(\rho_{\text {rel }}\right)_{0} a_{0}^{4} u^{4}+\left(\rho_{m}\right)_{0} a_{0}^{3} u^{3}\right]}}
$$

Applying Equation (11) to the denominator, and integrating leads to the following polynomial expression for $u=a^{-1}$

$$
u^{9}+A_{1} \cdot \frac{u^{8}}{a_{0}}+A_{2} \cdot \frac{u^{7}}{a_{0}^{2}}-A_{3} \cdot\left(\frac{\Lambda}{8 \pi}\right) \cdot \frac{u^{5}}{a_{0}^{4}}-A_{4} \cdot\left(\frac{\Lambda}{8 \pi}\right) \cdot \frac{u^{4}}{a_{0}^{5}}+A_{5} \cdot\left(\frac{\Lambda}{8 \pi}\right)^{2} \cdot \frac{u^{1}}{a_{0}^{8}}+A_{6} \cdot\left(\frac{\Lambda}{8 \pi}\right)^{2} \cdot \frac{t}{a_{0}^{9}} \cong 0
$$

We could go considerably higher in polynomial roots of Equation (12) above, depending upon the degree of accuracy we wished to obtain. This truncation so picked above is assuming a non infinite value of $u=a^{-1}$, as well as a non zero value, and non infinite value for the $\Lambda$ term. In doing so, we would obtain an extremely non standard evolution for the scale factor, assuming when we do so that

$$
A_{1}=\frac{9}{4} \cdot \frac{\left(\rho_{m}\right)_{0}}{\left(\rho_{\text {rel }}\right)_{0}}, \quad A_{2}=\frac{\left(\rho_{m}\right)_{0}^{2}}{\left(\rho_{\text {rel }}\right)_{0}^{2}}, \quad A_{3}=\frac{1 / 5}{\left(\rho_{\text {rel }}\right)_{0}^{1}}, \quad A_{4}=\frac{\left(\rho_{m}\right)_{0} \cdot(1 / 4)}{\left(\rho_{\text {rel }}\right)_{0}^{2}}, \quad A_{5}=\frac{1}{\left(\rho_{\text {rel }}\right)_{0}^{2}}, \quad A_{6}=\frac{1}{\left(\rho_{\text {rel }}\right)_{0}^{2}} \cdot \sqrt{\frac{\Lambda}{3}}
$$

If we go to Equation (9) above and assume small scale factor $a(t)$ values, as well as a not very large value for the $\Lambda$ term, we can approximate it by 


$$
\int \mathrm{d} a \frac{1}{\left[\frac{8 \pi}{\Lambda}\left[\left(\rho_{\text {rel }}\right)_{0} a_{0}^{4}+\left(\rho_{m}\right)_{0} a_{0}^{3} a(t)\right]\right]^{1 / 2}} \cong \sqrt{\frac{\Lambda}{3}} \cdot \int \mathrm{d} t
$$

This allows us to write a value of the scale factor along the lines of solving the equation of state of

$$
\left[\left(\frac{8 \pi}{\Lambda} \cdot\left[\left(\rho_{\text {rel }}\right)_{0} a_{0}^{4}+\left(\rho_{m}\right)_{0} a_{0}^{3} a(t)\right]\right)\right]^{1 / 2} \cong\left[\frac{\left(\rho_{m}\right)_{0} \cdot a_{0}^{3}}{2}\right] \cdot \frac{\Lambda}{\sqrt{24 \pi}} \cdot t
$$

This leads to the following value of the scale factor, namely for times slightly greater than values at the order of Planck's constant $t_{P}$

$$
a(t) \approx \frac{\Lambda^{3}}{768 \pi^{2}} \cdot\left(\rho_{m}\right)_{0} \cdot a_{0}^{3} \cdot t^{2}-\left[\frac{\left(\rho_{\text {rel }}\right)_{0}}{\left(\rho_{m}\right)_{0}}\right] \cdot a_{0}
$$

What is interesting in this situation is that this evokes a time dependence which is not dissimilar to the situation with the radiation dominated era, except that it is roughly slightly more than the square of the radiation dominated era. It is now time to consider what if we are looking at a situation where we have.

Equation (10) gives a homogenous solution with the following behavior for sufficiently small time intervals. This assumes of course that time is or the order of Planck's constant $t_{P}$

$$
a_{\text {General }} \sim a_{\text {initial }} \cdot \exp (i \cdot|\dot{H}| \cdot t) \Rightarrow \operatorname{Re}\left(a_{\text {General }}\right) \sim a_{\text {initial }} \cdot \cos (|\dot{H}| \cdot t) \sim a_{\text {initial }}
$$

For sufficiently large values of $|\dot{H}| \cdot t$, even if the time interval were extremely small, this would instead lead to the right hand side of the above equation tending to zero. Let us now look at what we can expect with respect to the particular equation solution and behavior of

$$
a^{4}-3 \cdot|\dot{H}| \cdot \frac{a^{2}}{\Lambda}+\left(\frac{8 \pi}{\Lambda}\right) \cdot\left[\left(\rho_{m}\right)_{0} \cdot a_{0}^{3} \cdot a+\left(\rho_{\text {rel }}\right)_{0} \cdot a_{0}^{4}\right] \equiv 0
$$

This is a non linear equation for the scale factor, to put it mildly. If we have that $a(t) \ll a_{0}$, and if $\left(\rho_{m}\right)_{0} \cdot a_{0}^{3} \cdot a+\left(\rho_{\text {rel }}\right)_{0} \cdot a_{0}^{4} \sim\left(\rho_{\text {rel }}\right)_{0} \cdot a_{0}^{4}$, then the above is approximated by

$$
a^{4}-3 \cdot|\dot{H}| \cdot \frac{a^{2}}{\Lambda}+\left(\frac{8 \pi}{\Lambda}\right) \cdot\left[\left(\rho_{\text {rel }}\right)_{0} \cdot a_{0}^{4}\right] \cong 0
$$

Leading to a value for the particular solution to Equation (10) for a scale factor for times in the neighborhood of the Planck constant value of

$$
a_{\text {Particular }} \sim \frac{1}{2} \cdot\left[\frac{3 \cdot|\dot{H}|}{\Lambda} \pm \sqrt{\frac{9 \cdot|\dot{H}|^{2}}{\Lambda^{2}}-\frac{32 \cdot \pi}{\Lambda} \cdot\left(\rho_{\text {rel }}\right)_{0} \cdot a_{0}^{4}}\right] \underset{\Lambda, \mid \dot{H} \rightarrow \text { Big }}{\longrightarrow} \frac{3 \cdot|\dot{H}|}{\Lambda} \neq 0
$$

Needless to say this would be particularly relevant to the solution of the initial value of the Cosmological constant parameter, as given by Barvinsky [3], i.e. that one would have for a maximum value of the cosmological constant greater than or equal to 316 times the value of the Planck mass $m_{P}$, squared, a situation where we would have an initial scale factor which is very small, but non zero. This of course would tend to the zero value if the cosmological constant would become significantly greater, i.e. to a temperature dependence as the initial temperature was of the order of $10^{32}$ Kelvin, that the particular solution of the scale factor would tend to zero.

\section{Appendix V: Forming the Fraction of a Critical Density, $\Omega_{G W}=\rho_{G W} / \rho_{C} \quad$ over the Logarithmic Interval of Frequency $\omega$}

We first of all start off with an estimate for a stringy value of the red shift, taking into account the transition from radiation dominance (in early universe conditions) to matter dominance. This entails setting $z_{e q} \sim 10^{4}$ as opposed to the CMB limiting value of $z_{C M B} \sim 10^{5}$ of about 1100 years after the big bang. Furthermore the ratio 
of the density of gravity waves

$\rho_{G W}$, over a critical density we call $\rho_{C}$ may be written as

$$
\Omega_{G W}=\rho_{G W} / \rho_{C}
$$

over frequency $\omega$, where one uses a scale expansion factor with respect to the dilaton scalar field $\phi$, with

$$
g=\exp (\phi / 2)
$$

In doing this, we also have to consider frequency ranges. The base line frequency range is set at about

$$
\omega_{1} \sim \text { upper bound of effective temperature } \approx 10^{11} \mathrm{~Hz}
$$

This is for the $Z_{e q} \sim 10^{4}$ red shift value, with a higher effective temperature expected when we look at $z_{C M B} \sim 10^{5}$. If we go 'backwards in time to the $z_{C M B} \sim 10^{5}$ value, we can expect that the $\omega_{1}$ maximum value will actually be higher, which will tend itself toward a different set of physical assumptions for what we need to work with for a differential ratio of $\mathrm{d} \Omega_{\text {GRW }} / \mathrm{d} \ln \omega \approx 10^{-10}$ as an upper limit to desired sensitivity for LIGO and other such instruments used to analyze gravitational wave/graviton physics. This will entail looking at the following

$$
\mathrm{d} \Omega_{G R W} / \mathrm{d} \ln \omega \equiv g_{1}^{2} \cdot z_{e q}^{-1} \cdot\left[\left(\frac{\omega}{\omega_{1}}\right)^{6-2 \beta}+\left(\frac{\omega}{\omega_{1}}\right)^{2 \beta}\right]
$$

This is for

$$
\beta=-\left(\log g_{s} / g_{1}\right) / \log z_{s}
$$

As I have been told by Dr. Puthoff in person [30], there is no reason why the value of the denominator in Equation (4) above could not be set at Planck's frequency value of $\omega_{1} \equiv 1.8551 \times 10^{43}$ per second which if we wished to have Equation (4) above as having an upper bound of $10^{-10}$, leaving a huge range of values of $\omega$ we can pick. By necessity, this leads us to calculate values of $\beta, g_{s}$, and $g_{1}$ for our estimation, which is what we will do, next. Here we can set for $\eta_{s} \propto-1, \quad z_{s} \approx 10^{10}$, and $\eta_{1} \propto-10^{-35}, z_{1} \approx 10^{27}$

$$
g_{s} \approx 1
$$

As well as

$$
g_{1}=\exp \left(\phi_{1} / 2\right) \approx \exp \left(-\frac{\sqrt{3}}{2} \cdot \log \left(-\eta_{1}=10^{-35}\right)\right) \approx 1.458 \times 10^{13}
$$

If $z_{1} \approx 10^{27} \cong z_{e q}$ we have that $g_{1}^{2} \cdot z_{\text {eq }}^{-1} \approx 0.1458$ and $\log z_{s} \approx 10$ and $\beta \approx 1.3$ leading to an effective value of, when $\omega_{1} \equiv 1.8551 \times 10^{43}$ a literally enormous number of frequency values for $\omega$

$$
\mathrm{d} \Omega_{G R W} /\left.\mathrm{d} \ln \omega\right|_{\text {effective }} \equiv 0.13 \times\left[\left(\frac{\omega}{\omega_{1}}\right)^{4.4}+\left(\frac{\omega}{\omega_{1}}\right)^{2.6}\right] \leq 10^{-10}
$$

\section{Appendix VI: Sach-Wolfe as a Function of CMB, and Gravitons Creating a CMB Profile}

The enormous number of frequency values permitted for relic Graviton production leads up to measurable consequences for photonic related CMB production. We will attempt to delineate what these are and to also look at when the interaction of gravitons commenced with early universe conditions to initiate CMB. Sach-Wolfe effect considerations are a way of quantifying the onset of large scale anisotropic contributions to variations of temperature picked up by CMBR measurements, and we will discuss their interaction.

We shall start off with Padmanabhan's temperature fluctuations of [29]

$$
\left[\frac{\Delta T}{T}\right]_{\text {Sach-Wolfe }}^{2} \propto \theta^{1-n}
$$


Here we have that the index of $n$ so written is usually greater than or equal to 2 leading to, for $\bar{\alpha} \geq 1$

$$
\left[\frac{\Delta T}{T}\right]_{\text {Sach-Wolfe }}^{1} \propto \theta^{-\bar{\alpha} / 2}
$$

With this in mid we really need to consider the role which back ground density variations play in the evolution of photonic contributions to CMBR. If we have a contribution of graviton frequency values with usually a strict inequality, and often a situation where $\omega \ll \omega_{\text {Planckian }}$ we can look at a time for when gravitons start to generate $\mathrm{CMB}$ contributions via photonic

$$
\left.\left[\frac{\Delta T}{T}\right]_{\text {Sach-Wolfe }}^{1} \sim\left\langle\frac{\delta \rho}{\rho}\right\rangle \sim \frac{3}{2 \cdot \sqrt{14}} \cdot t_{*}^{1 / 2}\right|_{\omega \ll \omega(\text { planckian })}
$$

So we have a threshold of when we have comparatively low frequency contributions of when gravitons start to contribute to CMBR as writable up as, for a set of values of $\theta \cong 180 / l$. This would be for $z_{\text {Planck }} \approx 10^{27}>z_{*} \geq z_{1-\text { sec }} \approx 10^{10}$ and low to middle value of $\bar{\alpha}$, and low values of $l$.

$$
\left.t_{*}\right|_{\omega \ll \omega(\text { Planckian })} \sim \frac{59}{9} \cdot\left[\frac{l^{\bar{\alpha}}}{(180)^{\bar{\alpha}}}\right] \sim 1 \text { second up to values of one minute }
$$

If we have a situation where the ratio of graviton frequency as given by $x=\omega /(T=2.7$ Kelvin $)$ is large, as the frequency approaches a high Planck frequency value, we obtain, instead

$$
\left.t_{*}\right|_{\omega \leq \omega \text { (PLanckian) }} \sim \frac{1}{x} \frac{59}{9} \cdot\left[\frac{l^{\bar{\alpha}}}{(180)^{\bar{\alpha}}}\right] \sim 1 / x \ll 1 \text { second. }
$$

We can then for frequencies far larger than what we would expect for the typical values of the LISA experiment see

$$
\left.t_{*}\right|_{\omega \leq \omega(\text { PLanckian })} \sim 10^{-35} \approx t_{\text {Planck }}
$$

i.e. the beginning of interaction of the gravitons with early universe conditions would be at the onset of the inflationary era. Big surprise, NOT.

We should compare this set of estimates for more traditional bounds for the onset of nucleosynthesis in the early universe as given by Dodelson [31] in his book "Modern Cosmology". As an example, we find that the onset of when deuterium and other light elements are created after the big bang can be predicted via, for $T \sim 0.07 \mathrm{MeV}$ such that after about two minutes deuterium begins to form

$$
t \cong(132 \mathrm{sec}) \times\left(\frac{0.1 \mathrm{MeV}}{T}\right)^{2}
$$

This also, after we go from a red shift of $z \approx 10^{10} \rightarrow z \approx 1100$, for $T \sim 0.25 \mathrm{eV}$ we get Equation (7) of the order of $t \cong 2.1 \times 10^{13}$ seconds for when photons of CMBR decouple from matter. Seen from this perspective we can say that the onset of graviton production as interacting with early universe conditions for relic CMB data "traces" at the very least is initiated about a full minute before light element production commences. The estimates given by Equation (5) and Equation (6) point to this occurring as early as a Planck time interval of time.

In order to make sense of what Equation (5) and Equation (6) are telling us, we need to among other things consider initial energy density values which are relativistic in nature. Dodelson [31] writes a first order baryonic approximation of critical density values via considering the effective number of relativistic degrees of freedom $g_{*}$ which is in early universe conditions of about 3.36 in value, as of about the first minute of creation after the big bang [31].

$$
\rho_{\text {relativistic-matter,energy }} \equiv g_{*} \cdot \frac{\pi^{2}}{30} \cdot T^{4}
$$

This can be compared with Dodelson's [31] critical energy value of about $8.098 h^{2} \times 10^{-11} \mathrm{eV}^{4} /(\hbar \cdot c)^{3}$ which means that we need something other than the effective number of relativistic degrees of freedom $g_{*}$ and de coupling of photons (light) from matter as a way to consider the onset of graviton production as a way of modeling the Sach-Wolfe contribution to large scale anisotopic background in CMB. To do this, we will consider the 
influence of relic electromagnetic fields, in particular, magnetic fields to the problem of how gravitons affect CMBR in the early onset of inflation.

It is important to note that Pisen Chen [32] as of 1994 denoted the onset of graviton interaction with the generation of a huge gravitation surge with a relic magnetic field. His value of that magnetic field was $B_{\text {relic }} \leq 10^{11}$ Gauss, for graviton interactions with early universe "matter" at about one minute of time after the big bang. This is in contrast to the value of that initial magnetic field as allegedly being of the form $B_{\text {today }} \leq 10^{-7}$ Gauss as measurable via space craft instrumentation in today's astrophysical data collection milieu.

What Pisen Chen gives us in his 1994 paper is a way to tie in the purported value of the relic magnetic field, as proportional to temperature fluctuations, as given by the following upper bound inequality. This is for the Planck "constant value" $H_{1}$ left as an "open parameter" but understood as being set at the ONSET of inflation, $\omega_{0}$ being the initial frequency of a graviton production process, and $\delta \Omega_{E M} \sim \frac{B_{*}^{2}}{8 \pi} \cdot \frac{1}{\rho_{C}^{*}}$ as well as $T_{\text {present }} \sim 2.7 \mathrm{~K}$

$$
\left[\frac{\sinh ^{2}\left(\omega_{0} / T_{\text {present }}\right)}{\left(\omega_{0} / T_{\text {present }}\right)}\right] \cdot\left[\frac{4 \pi^{4}}{15}\right] \cdot\left[\frac{10^{-29} \times T_{\text {present }}}{H_{1}}\right]^{4} \cdot \delta \Omega_{E M}^{*} \leq\left\langle\frac{\delta T}{T}\right\rangle
$$

If the ratio of $\omega_{0}$ to $T_{\text {present }} \sim 2.7 \mathrm{~K}$ is large, this means that

$$
\delta \Omega_{E M} \sim \frac{B_{*}^{2}}{8 \pi} \cdot \frac{1}{\rho_{C}^{*}} \sim\left\langle\frac{\delta T}{T}\right\rangle
$$

To get an idea of what this is saying, we can state that for large anisotropic variations in CMB we have for even strong initial magnetic fields a much larger value of the critical density of early space time matter-energy as given by the denominator of the left hand side of Equation (10) above at even the one minute or so mark for times after the big bang. This would lead to us asking how we could determine both the relic magnetic field $B_{*}$ as well as the critical density $\rho_{C}^{*}$. Furthermore, if the * label means the values of both the magnetic field and of density at the onset of the beginning of gravitons starting to interact with space time to produce the Sach-Wolfe CMBR signatures are such that for near relic graviton conditions we have a much, much larger $\rho_{C}^{*}$ value, albeit tending to almost being of infinite value as we push the onset of graviton creation of CMBR photonic traces closer and closer to the Planck time value of $t_{P} \sim 10^{-35}$ seconds. Interestingly enough, this Equation (10) holds even if we go to Pisen Chen's [32] value of frequency as allegedly predicted by string theory, i.e.

$$
\omega_{0} \approx 10^{29} \cdot \sqrt{H_{0} / M_{P}} \cdot\left(\omega_{1} \sim 10^{-18}\right) \text { Hertz } \sim 10^{11} \text { Hertz }
$$

\section{Appendix VII: A Working Mathematical Model for Short Term Quintessence, up to a Planck Interval of Time}

\section{CASE I:}

Temperature $T$ very small, a.k.a. Carroll and Chen's [20] [21] suppositions and time less than $t_{P}$. This is the slow roll case, which is also true when we get to time $\gg t_{P} t_{P}$

$$
\begin{aligned}
& \ddot{\phi} \cdot\left[1+\frac{\tilde{c}}{M^{2}} \cdot \frac{T^{2}}{6} \cdot g_{b}\right]+3 \cdot H \cdot \dot{\phi} \cdot\left[1+\frac{\tilde{c}}{M^{2}} \cdot \frac{T^{2}}{6} \cdot g_{b}\right]+\left(\frac{\partial V_{\text {axion-contri }}}{\partial \phi}\right) \\
& \underset{T \rightarrow 0^{+}}{\longrightarrow} 3 \cdot H \cdot \dot{\phi}+\left(\frac{\partial V_{\text {axion-contri }}}{\partial \phi}\right) \cong 0
\end{aligned}
$$

CASE II:

Temperature $T$ very large and time in the neighborhood of $t_{P}$. This is NOT the slow roll case, and has $H \propto 1 / t_{P}$. Note, which is important that the constant $c$ is not specified to be a small quantity

$$
\begin{aligned}
& \ddot{\phi} \cdot\left[1+\frac{\tilde{c}}{M^{2}} \cdot \frac{T^{2}}{6} \cdot g_{b}\right]+3 \cdot H \cdot \dot{\phi} \cdot\left[1+\frac{\tilde{c}}{M^{2}} \cdot \frac{T^{2}}{6} \cdot g_{b}\right]+\left(\frac{\partial V_{\text {axion-contri }}}{\partial \phi}\right) \\
& \underset{T \rightarrow 10^{12} \mathrm{Kelvin}}{\longrightarrow} \ddot{\phi}+3 \cdot H \cdot \dot{\phi}+\left(\frac{\tilde{c}}{M^{2}} \cdot \frac{T^{2}}{6} \cdot g_{b}\right)^{-1} \cdot\left(\frac{\partial V_{\text {axion-contri }}}{\partial \phi}=m^{2} \cdot\left(\phi-\phi_{C}\right)\right) \cong 0
\end{aligned}
$$


We then get a general, and a particular solution with $\phi_{\text {general }} \propto \exp (p \cdot t), \phi_{\text {particular }} \equiv \phi_{C}, \phi_{\text {Total }}=\phi_{\text {general }}+\phi_{\text {particular }}$,

$$
\begin{aligned}
& p^{2}+3 \cdot H \cdot p+\left(\frac{\tilde{c}}{M^{2}} \cdot \frac{T^{2}}{6} \cdot g_{b}\right)^{-1} \cdot\left(m^{2}\right) \cong 0 \\
& \rightarrow p \cong\left[-\frac{3 H}{2} \cdot\left[2-4 \cdot \frac{m^{2} \cdot M^{2}}{T^{2} \cdot c \cdot g_{b} H}\right],-\left(6 \cdot \frac{m^{2} \cdot M^{2}}{T^{2} \cdot c \cdot g_{b}}\right) \approx-\varepsilon^{+}\right] \equiv\left[p_{1}, p_{2}\right] \\
& \Rightarrow \phi_{\text {general }} \cong c_{1} \cdot \exp \left(-\left|p_{1}\right| \cdot t\right)+c_{2} \cdot \exp \left(-\left(\left|p_{2}\right| \approx \varepsilon^{+}\right) \cdot t\right) \\
& \quad \phi_{\text {Total }}=\phi_{\text {general }}+\phi_{\text {particular }} \cong \phi_{C}+\varepsilon_{1} \cdot \phi_{\text {initial value }}+\text { H.O.T. }, \text { where } \varepsilon_{1}<1
\end{aligned}
$$

CASE III:

Temperature $T$ very large and time in the neighborhood of $t_{p}$. This is NOT the slow roll case, and has $H \propto 1 / t_{p}$. Note, which is important that the constant $c$ IS specified to be a small quantity. We get much the same analysis as before except the higher order terms (H.O.T.) do not factor in

$$
\phi_{\text {Total }}=\phi_{\text {general }}+\phi_{\text {particular }} \cong \phi_{C}+\varepsilon_{1} \cdot \phi_{\text {initial value }} \text {, where } \varepsilon_{1}<1
$$

Case IV:

Temperature $T$ not necessarily large but on the way of becoming large valued, so the axion mass is not negligible, YET, and time in the neighborhood of $t_{P}$. This is NOT the slow roll case, and has $H>H_{t=t_{P}} \propto 1 / t_{P}$. Begin with making the following approximation to the Axion dominated effective potential

$$
\begin{aligned}
& V_{\text {axion-contri }} \equiv f\left[m_{\text {axion }}(T)\right] \cdot(1-\cos (\phi))+\frac{m^{2}}{2} \cdot\left(\phi-\phi_{C}\right)^{2} \\
& \Rightarrow\left(\frac{\partial V_{\text {axion-contri }}}{\partial \phi}\right) \frac{}{\text { Temperature getting larger }} \\
& f\left[m_{\text {axion }}(T)\right] \cdot \frac{\phi^{5}}{125}-f\left[m_{\text {axion }}(T)\right] \cdot \frac{\phi^{3}}{6}+\left[\left(m^{2}+f\left[m_{\text {axion }}(T)\right]\right) \cdot \phi-m^{2} \phi\right]
\end{aligned}
$$

Then we obtain

$$
\ddot{\phi}+3 \cdot H \cdot \dot{\phi}+\left(\frac{\tilde{c}}{M^{2}} \cdot \frac{T^{2}}{6} \cdot g_{b}\right)^{-1} \cdot\left(\frac{\partial V_{\text {axion-contri }}}{\partial \phi}\right) \cong 0
$$

This will lead to as the temperature rises we get that the general solution has definite character as follows

$$
\begin{aligned}
& p^{2}+3 \cdot H \cdot p+\left(\frac{\tilde{c}}{M^{2}} \cdot \frac{T^{2}}{6} \cdot g_{b}\right)^{-1} \cdot\left(m^{2}\right) \cong 0 \\
& \rightarrow p \cong\left[-\frac{3 H}{2} \cdot\left[1 \pm \sqrt{1-\frac{6 \cdot M^{2}}{3 \cdot T^{2} \cdot c \cdot g_{b} H} \cdot\left(m^{2}+f\left[m_{\text {axion }}(T)\right]\right)}\right]\right] \equiv\left[p_{1}, p_{2}\right] \\
& \Rightarrow \phi_{\text {general }} \cong c_{1} \cdot \exp \left(p_{1} \cdot t\right)+c_{2} \cdot \exp \left(p_{2} \cdot t\right) \\
& \propto[\phi(\text { real })+i \cdot \phi(\text { imaginary })] \text { iff }\left[m_{\text {axion }}(T)\right] \text { large } \\
& \propto[\phi(\text { real })] \text { iff }\left[m_{\text {axion }}(T)\right] \text { small }
\end{aligned}
$$

The upshot is, that for large, but shrinking axion mass contributions we have a cyclical oscillatory system, which breaks down and becomes a real field if the axion mass disappears. First of all, though, we have to understand how the conditions presented by S. Carroll, and J. Chen [20] [21] came about via brane theory. Our point is that we need brane theory to establish the initial starting point of reference for low temperature conditions at the onset of inflation. 


\section{Submit or recommend next manuscript to SCIRP and we will provide best service for you:}

Accepting pre-submission inquiries through Email, Facebook, Linkedin, Twitter, etc A wide selection of journals (inclusive of 9 subjects, more than 200 journals)

Providing a 24-hour high-quality service

User-friendly online submission system

Fair and swift peer-review system

Efficient typesetting and proofreading procedure

Display of the result of downloads and visits, as well as the number of cited articles

Maximum dissemination of your research work

Submit your manuscript at: http://papersubmission.scirp.org/ 